\title{
TEVATRON I
}

\section{SMALL APERTURE QUAD LAMINATION ANALYSIS}

Mark Leininger

September 24,1982

\begin{abstract}
Stacking, compression, and welding of the laminations for the TeV I Small Aperture Quad results in a deformation due to springback which is unacceptable due to magnetic field requirements. ANSYS has been used to analyze a solution to this problem.
\end{abstract}

\section{Introduction}

Fig. 1 and 2 show the geometry of the model used to analyze the quad lamination problem. The model represents one half of a lamination. The stacking and compression of the laminations subject them to a pressure in the $y$ direction of 200 psi. After welding the laminations together the compressive force is released, resulting in a springback in the area of the poleface (the piece that looks like a tongue). One obvious solution is to machine a solid steel plate for each end of the lamination stack which would resist this springback. This is a very costly proposition as it turns out the plate needs to be about 5 to 6 in. thick. Joe Heim proposed the idea to epoxy the last $n$ laminations together so they will act like a solid plate without 
the expense of a solid plate. The object of this analysis is to determine $n$, that is, how many $1 / 16$ in. thick steel laminations must be epoxied together to result in about a .005 to .010 in. deflection near the poleface and have an acceptable shear stress between laminations.

\section{Solution}

The analysis was performed using $3 \mathrm{D}$ solid elements to allow in plane shear information to be extracted. The analysis was performed using three elements through the thickness of the epoxied assembly (Fig. 2) for overall thicknesses of 2 in., 4 in., and 6 in. The support constraints are shown in Fig. 3. The lamination has UY constraints (constrained against translation in the $y$ direction) at each place where a steel bar will be welded along the outer edge of the laminations. This represents a simple support condition. Along the symmetry axis, rotation is prevented by applying UZ constraints through the thickness which is equivalent to requiring that the slope be zero. A summary of the results is shown in Table 1. It appears that a 6 in. thickness will satisfy the deflection and shear stress requirements. A distorted geometry plot of the 2 in. plate is shown in Fig. 4 . 


\section{Table I}

Quad Lamination Summary

Overall

$$
\text { Maximum } Y
$$

Thickness (in.)

Deflection (in.)

$$
0.084
$$

0.016

0.006
TYZ (psi)

6452

2408

1292
$\underline{\mathrm{XZ} \text { (psi) }}$ 6660

2147

1058

\section{Assumptions and Limitations}

The model used to solve this problem is quite a simplification of the real case. The stack of epoxied laminations not only has a different stiffness than the solid plate assumed in this model, but is really anisotropic. The modulus in the $y$ direction will be modified by the epoxy between layers, but the $\mathbf{x}$ and $z$ moduli are essentially the same as for steel. It is difficult to guess the magnitude of these effects, but they probably are not greater than 10 or 20 percent. The author feels the model used is sufficient to give the required accuracy. 
Fig. 1

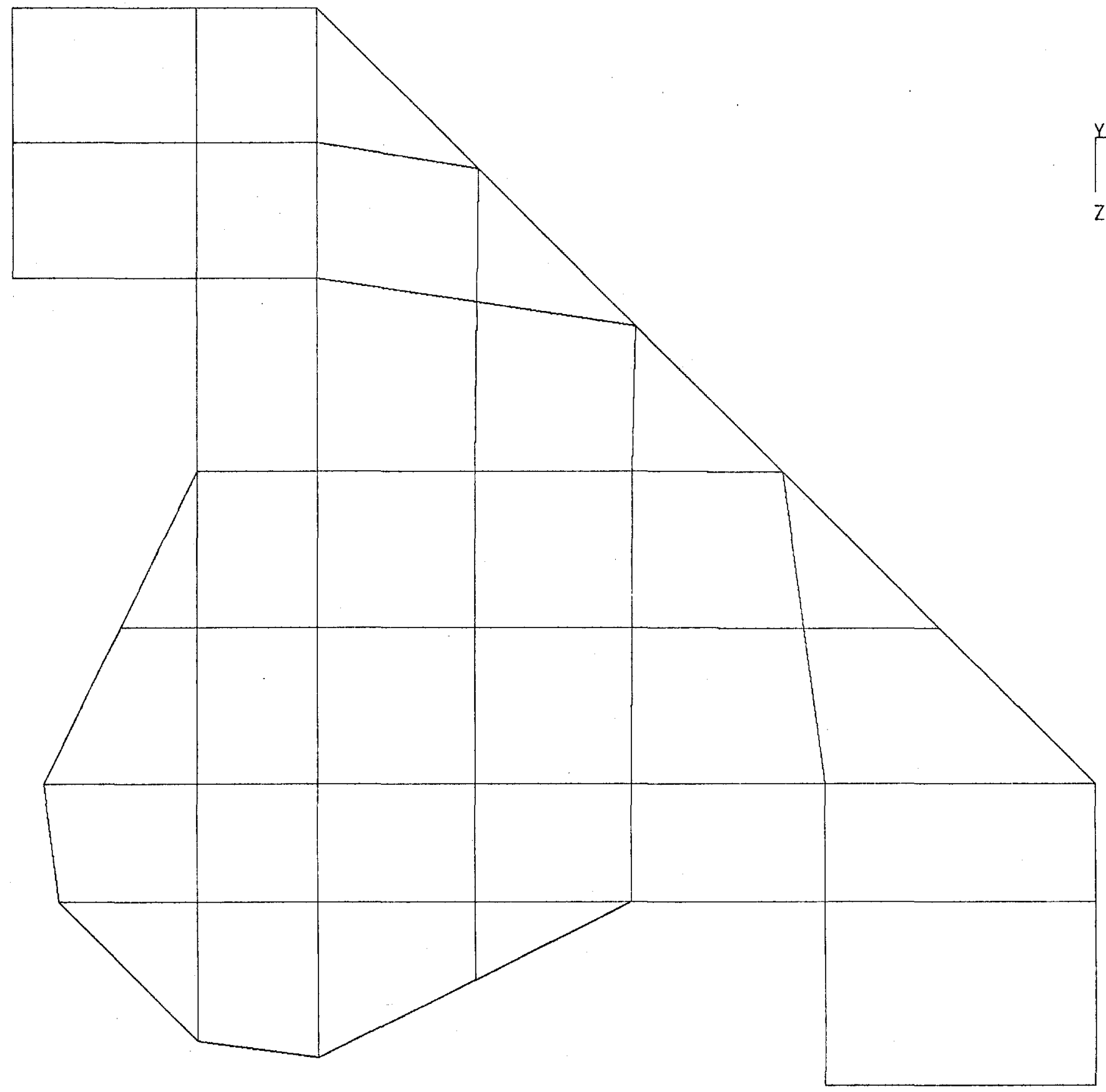


Fig. 2

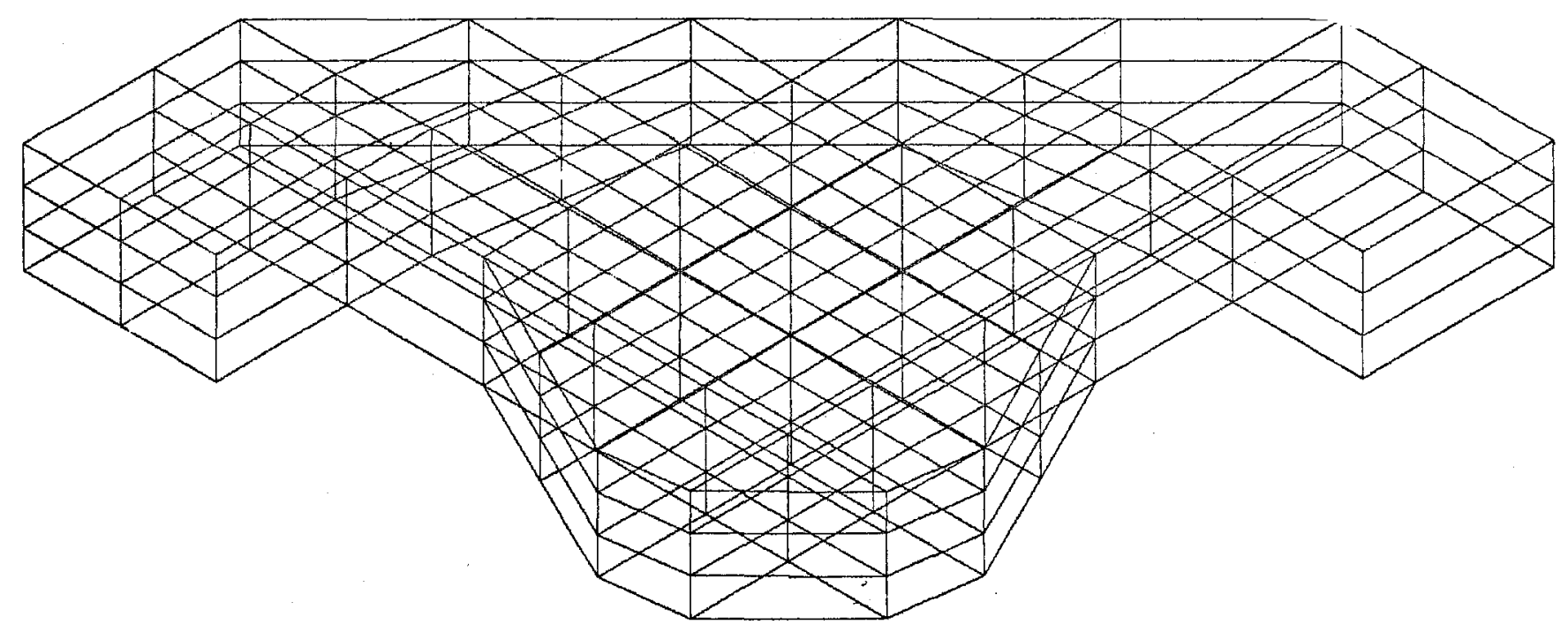


Fig. 3

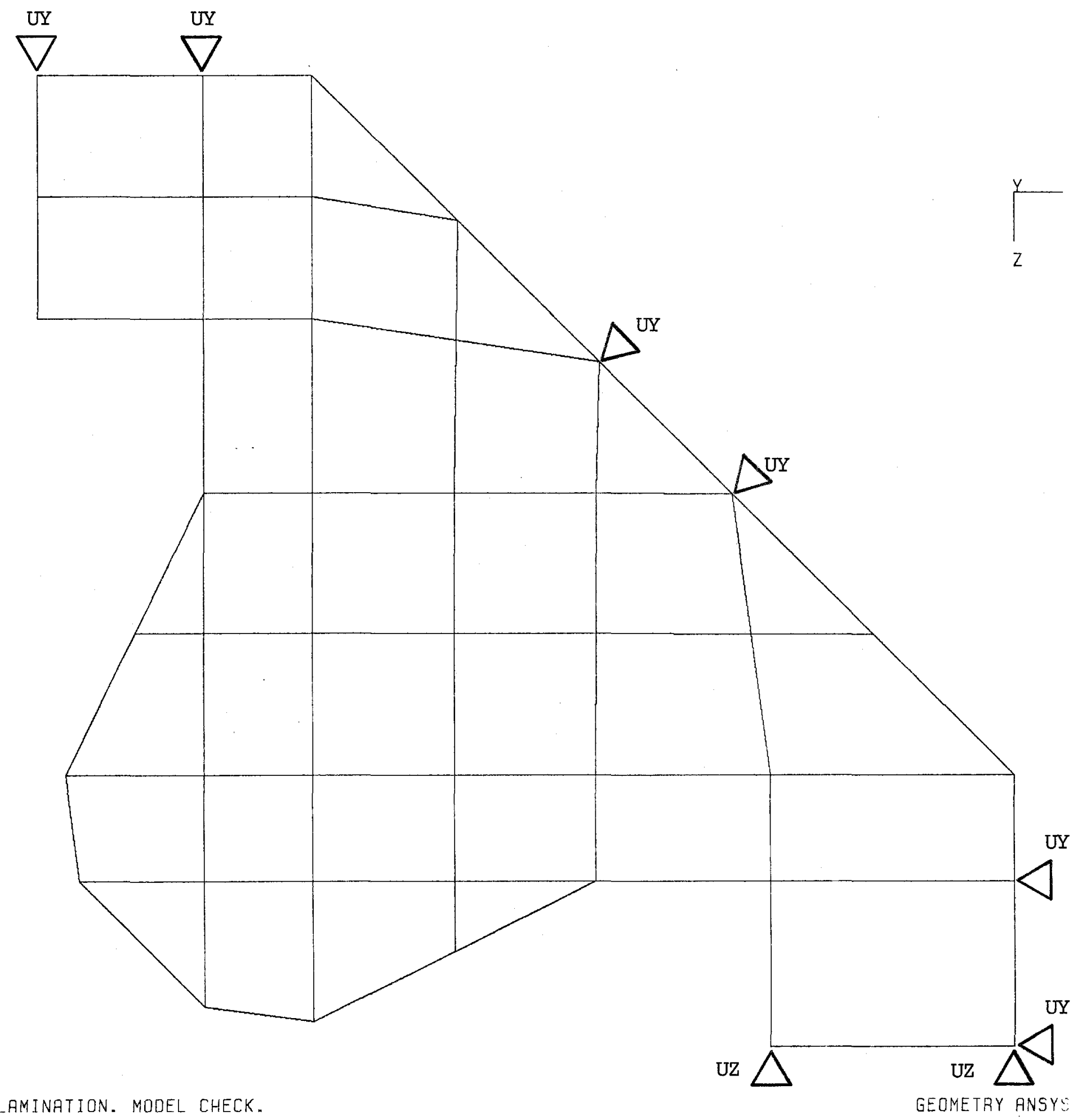


Fig. 4

STEP $=1$ ITER $=1$ TIME $=0$

$.0841 \varepsilon$
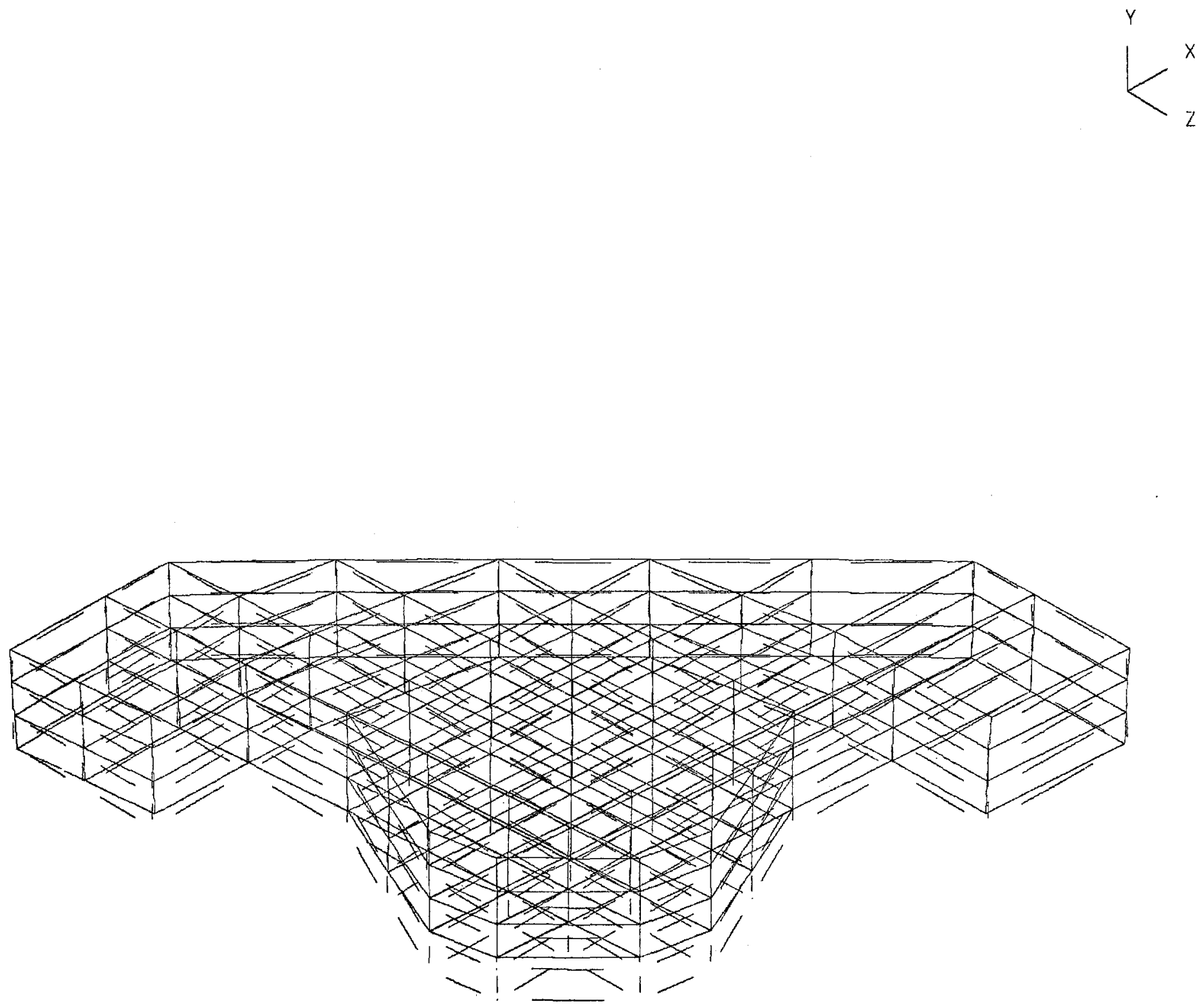